\title{
Avaliação da influência hormonal em pacientes com fraturas atribuídas a osteoporose*
}

\section{Evaluation of Hormonal Influence in Patients with Fractures Attributed to Osteoporosis}

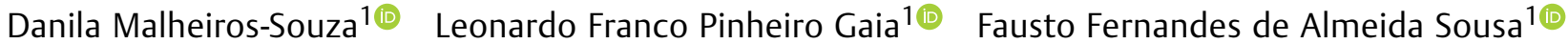 \\ Pedro Ivo Ferreira Favaro ${ }^{1}$ () Virmondes Rodrigues ${ }^{1(0)}$ Denise Bertulucci Rocha Rodrigues ${ }^{1,2(1)}$
}

1 Departamento de Ciências Biológicas, Laboratório de Imunologia,
Universidade Federal do Triângulo Mineiro, Uberaba, MG, Brasil
2 Laboratório de Imunobiologia, Universidade de Uberaba, Uberaba, MG, Brasil

Endereço para correspondência Denise Bertulucci Rocha Rodrigues, PhD, Universidade de Uberaba, Uberaba, MG, Av Nenê Sabino, 1801. Uberaba MG, 38055-500, Brasil (e-mail: denise.rodrigues@uniube.br).

Rev Bras Ortop 2021;56(6):804-808.

\begin{abstract}
Resumo
Objetivo Avaliar a influência dos níveis hormonais de vitamina $D$, calcitonina, testosterona, estradiol e paratormônio em pacientes com fratura atribuída a osteoporose, quando comparados com pacientes jovens que tiveram fraturas decorrentes de acidente de alto impacto.

Métodos Foram coletadas amostras de sangue de 30 pacientes idosos com fratura atribuída a osteoporose (T-score $\leq-2,5$ ) (grupo com osteoporose) e 30 amostras de sangue de pacientes jovens que sofreram fraturas decorrentes de acidentes de alto impacto (grupo controle). Foram realizadas dosagem de 1,25-hidroxivitamina D (Kit Diasorin, Saluggia, Italy), calcitonina (Kit Siemens, Tarrytown, NY, USA), testosterona, estradiol e paratormônio (Kit Beckman Couter, Indianapolis, IN, United States) pela técnica de quimiluminescência. Os dados foram inseridos em uma planilha de dados no programa Microsoft Excel (Microsoft Corp., Redmond, WA, EUA) e analisados pelo programa de estatística Statview. Os resultados que apresentaram distribuição não normal foram analisados com métodos não paramétricos. Para análise de variáveis comparando-se os dois grupos, aplicou-se o teste Mann-Whitney. Foi

Palavras-chave

- osteoporose

- hormônios

- estradiol

- calcitonina

- hormônio paratireóideo utilizado o teste de correlação de Spearman para a correlacionar os níveis hormonais. Um valor-p $>0.05$ foi considerado significante. Todas as análises foram feitas comparando gênero e grupos de pacientes com e sem osteoporose.

Resultados Mulheres com osteoporose apresentam níveis significativamente menores de estradiol e vitamina $\mathrm{D}(p=0.047$ e $p=0.0275)$, respectivamente. Homens com osteoporose demonstraram níveis significativamente maiores de paratormônio $(p=0.0065)$. Não houve diferença significativa nos níveis de testosterona e calcitonina.
\end{abstract}

Trabalho desenvolvido no Laboratório de Imunologia, Departamento de Ciências Biológicas, Universidade Federal do Triângulo Mineiro, Uberaba, MG, Brasil. recebido

05 de Agosto de 2020

aceito

01 de Dezembro de 2020

Publicado on-line

Agosto 27, 2021
DOI https://doi.org/ 10.1055/s-0041-1726065. ISSN $0102-3616$.
(C) 2021. Sociedade Brasileira de Ortopedia e Traumatologia. All rights reserved.

This is an open access article published by Thieme under the terms of the Creative Commons Attribution-NonDerivative-NonCommercial-License, permitting copying and reproduction so long as the original work is given appropriate credit. Contents may not be used for commercial purposes, or adapted, remixed, transformed or built upon. (https://creativecommons.org/ licenses/by-nc-nd/4.0/)

Thieme Revinter Publicações Ltda., Rua do Matoso 170, Rio de Janeiro, RJ, CEP 20270-135, Brazil 


\section{Abstract}

\author{
Keywords \\ - osteoporosis \\ - hormones \\ - estradiol \\ - calcitonin \\ - parathyroid hormone
}

Conclusão Existem diferenças hormonais entre os gêneros na osteoporose. Em mulheres, níveis significativamente menores de estradiol e vitamina D e, nos homens, níveis significativamente maiores de paratormônio, parecem influenciar na doença. Objective The present study aims to evaluate the influence of hormonal levels of vitamin $\mathrm{D}$, calcitonin, testosterone, estradiol, and parathyroid in patients with fractures attributed to osteoporosis when compared with young patients with fractures resulting from high-impact accidents.

Methods Blood samples were collected from 30 elderly patients with osteoporosisattributed fractures (T-score $\leq-2.5$ ) (osteoporotic group), and from 30 young patients with fractures resulting from high-impact accidents (control group). Measurement of 1,25hydroxyvitamin D (Kit Diasorin, Saluggia, Italy), calcitonin (Kit Siemens, Tarrytown, NY, USA), testosterone, estradiol, and parathyroid hormone (Kit Beckman Couter, Indianapolis, IN, United States) was performed using a chemiluminescence technique. Data were inserted into a Microsoft Excel (Microsoft Corp., Armonk, WA, USA) spreadsheet and analyzed using Statview statistical software. Results showing non-normal distribution were analyzed with nonparametric methods. The Mann-Whitney test was applied for group comparison, and a Spearman test correlated hormonal levels. Statistical significance was set at $p<0.05$. All analyzes compared gender and subjects with and without osteoporosis.

Results Women with osteoporosis had significantly lower levels of estradiol and vitamin $\mathrm{D}(p=0.047$ and $p=0.0275$, respectively). Men with osteoporosis presented significantly higher levels of parathyroid hormone $(p=0.0065)$. There was no significant difference in testosterone and calcitonin levels.

Conclusion Osteoporosis patients presented gender-related hormonal differences. Women had significantly lower levels of estradiol and vitamin D, whereas men had significantly higher parathyroid hormone levels, apparently impacting the disease.

\section{Introdução}

A osteoporose é causada por um desequilíbrio no metabolismo ósseo normal entre os osteoblastos e osteoclastos, ${ }^{1}$ sendo que, no processo de reabsorção óssea, o osteoclasto parece ter um papel mais ativo em relação ao osteoblasto. ${ }^{2,3}$ Estudos apontam que hormônios podem ter um papel importante no desequilíbrio de formação óssea ${ }^{4}$, visto que o paratormônio parece induzir o osteócito a diferenciar em osteoclasto. ${ }^{5}$ Da mesma forma, hormônios como o estradiol e a testosterona parecem ser importantes, ${ }^{6-8}$ uma vez que a testosterona age inibindo a apoptose de osteoblastos. ${ }^{9}$ Já o estrogênio parece ter um papel importante na remodelação óssea, tanto em homens quanto em mulheres, uma vez que esse hormônio parece estimular a liberação de calcitonina, além de ativar receptores de vitamina D no intestino, permitindo sua função endócrina e imunológica no processo do metabolismo ósseo. ${ }^{10,11}$ A calcitonina e a vitamina D ajudam a manter concentrações adequadas de cálcio no soro para permitir a mineralização normal do osso; além disso, a vitamina $D$ é necessária para o crescimento ósseo e a remodelação óssea por osteoblastos e osteoclastos. ${ }^{12}$

Com o aumento da expectativa de vida, doenças associadas à mudança hormonal, como a osteoporose, estão aumentando em quantidades consideráveis; por isso, a importância do foco em estudos com a finalidade de tornar esta interação mais clara.

O presente estudo tem como objetivo avaliar a influência dos níveis hormonais de vitamina $\mathrm{D}$, calcitonina, testosterona, estradiol e paratormônio em pacientes com fratura atribuída a osteoporose, quando comparados a pacientes jovens que tiveram fraturas decorrentes de acidente de alto impacto.

\section{Materiais e métodos}

O projeto foi aprovado pelo Comitê de Ética com protocolo de número 51827515.4.0000.5145.

\section{Grupo de Estudo}

Foram coletadas amostras de sangue de 30 pacientes idosos com fratura atribuída à osteoporose (T-score $\leq-2,5$ ) (grupo com osteoporose) e 30 amostras de sangue de pacientes jovens que sofreram fraturas decorrentes de acidentes de alto impacto (grupo controle). Foram excluídos os pacientes com outras doenças ósseas, com fraturas não causadas por osteoporose, pacientes imunossuprimidos, com neoplasias malignas ou alterações hepáticas, e que não concordaram participar da pesquisa. O soro coletado desses pacientes foi utilizado para dosagem hormonal.

\section{Coleta de sangue}

A coleta de sangue venoso foi realizada sempre no período matutino, 1 dia após a cirurgia de reconstrução óssea indicada pelo médico ortopedista. A amostra de sangue foi obtida 
por punção venosa, com a utilização de três tubos de coleta a vácuo contendo ativador de coágulo e gel separador. Após 30 minutos da coleta, foi feita a centrifugação a 5.000 rotações por minuto (rpm) por 10 minutos para obtenção de soro.

\section{Análise hormonal}

Após a centrifugação das amostras de sangue coletadas, os tubos de soro foram encaminhados para análise hormonal. Foram realizadas dosagem de 1,25-hidroxivitamina D (Kit Diasorin, Saluggia, Italy), calcitonina (Kit Siemens, Tarrytown, NY, USA), testosterona, estradiol e paratormônio (Kit Beckman Couter, Indianapolis, IN, United States) pela técnica de quimiluminescência, uma reação química, a qual, ao se processar, gera energia luminosa. Durante a reação, os reagentes se transformam em estados intermediários eletronicamente excitados e, ao passarem para um estado de menor excitação, liberam a energia absorvida na forma de luz.

\section{Análise estatística}

Os dados foram inseridos em uma planilha de dados no programa Microsoft Excel (Microsoft Corp., Redmond, WA, EUA) e analisados pelo programa de estatística Statview. Os resultados que apresentaram distribuição não normal foram analisados com métodos não paramétricos. Para análise de variáveis comparando-se os dois grupos, aplicou-se o teste Mann-Whitney. Foi utilizado o teste de correlação de Spearman para a correlacionar os níveis hormonais. Um valor$\mathrm{p}<0,05$ foi considerado significante.

\section{Resultados}

No presente estudo, foi realizada a dosagem hormonal de 60 pacientes, sendo 30 de pacientes com osteoporose e 30 do grupo controle.

A média de idade dos pacientes foi de 58,8 $\pm 22,61$ anos, e todas as análises foram feitas comparando gênero, grupo de pacientes com osteoporose e grupo controle. Os dados com o número de pacientes e a média de idade de cada grupo estão contidos na - Tabela 1.

Os níveis séricos de vitamina $\mathrm{D}$ foram significativamente maiores no gênero feminino do grupo controle quando comparados com o gênero masculino do grupo controle (Mann Whitney; $p=0.0169$ ). Ao comparar os níveis séricos de vitamina D entre os dois grupos (controle e osteoporose), observou-se níveis significativamente maiores no gênero feminino do grupo controle quando comparadas com o gênero feminino do grupo com osteoporose $(p=0.0275)$. Não houve diferença significativa entre os gêneros masculinos e femininos do grupo com osteoporose (-Figura 1A).

$\mathrm{Na}$ análise de testosterona livre, foram observados níveis significativamente maiores no gênero masculino quando comparado com gênero feminino em ambos os grupos, controle e osteoporose (Mann Whitney; ${ }^{*} \mathrm{p}=0.0023 \mathrm{e}^{* *} \mathrm{p}$ $=0.0046$ ). Não houve diferença significativa nos níveis de testosterona livre entre o gênero masculino do grupo controle e do grupo com osteoporose (-Figura 1B).

Os níveis de estradiol foram significativamente menores nas mulheres do grupo com osteoporose comparados com as do grupo controle (Mann-Whitney; $p=0.047$ ). Não houve diferença significativa nos níveis de estradiol entre homens e mulheres do grupo controle e do grupo com osteoporose (- Figura 1C).

Os níveis de paratormônios foram significativamente maiores nos homens com osteoporose quando comparados com o grupo controle (Mann-Whitney; $p=0.0065$ ). Não houve diferença significativa entre as mulheres do grupo controle com as mulheres do grupo com osteoporose. Tampouco foi observada uma diferença significativa nos níveis de paratormônios quando comparados os gêneros masculino e feminino em ambos os grupos, controle e osteoporose (-Figura 1D).

Não houve diferença significativa nos níveis de calcitonina entre o grupo controle e o grupo com osteoporose, independentemente do gênero. Também não houve diferença significativa quando comparados homens e mulheres independentemente dos grupos, controle e osteoporose (-Figura 1E).

\section{Discussão}

A osteoporose é causada por um desequilíbrio da remodelação óssea, que pode ser causado por fatores hormonais; além disso, estudos mais recentes mostram que fatores imunológicos também influenciam na fisiopatogênese da doença. No presente estudo, foi avaliada a presença de hormônios em pacientes com osteoporose, comparando também a diferença entre os gêneros.

No presente estudo, diferenças significativas foram encontradas nas dosagens de vitamina $\mathrm{D}$, estradiol e paratormônio. Mulheres com osteoporose e homens jovens apresentaram níveis significativamente menores de vitamina D comparados com mulheres jovens. Estudos mostram que a deficiência de vitamina D está associada a fraqueza muscular, perda óssea, quedas e fraturas. ${ }^{13}$ Semelhante à literatura, nossos dados sugerem que a diminuição de vitamina D em

Tabela 1 Distribuição da média de idade dos pacientes (mínima e máxima) em números absolutos, de acordo com o gênero feminino e masculino do grupo controle e do grupo com osteoporose

\begin{tabular}{|l|l|l|l|l|}
\hline & $\begin{array}{l}\text { Média idade } \\
\text { (anos) }\end{array}$ & $\begin{array}{l}\text { Número de } \\
\text { pacientes (n) }\end{array}$ & $\begin{array}{l}\text { Idade mínima } \\
\text { (anos) }\end{array}$ & $\begin{array}{l}\text { Idade máxima } \\
\text { (anos) }\end{array}$ \\
\hline Controle feminino & 39,5 & 08 & 18 & 58 \\
\hline Controle masculino & 39,6 & 22 & 19 & 58 \\
\hline Osteoporose feminino & 80,05 & 18 & 64 & 98 \\
\hline Osteoporose masculino & 74,9 & 12 & 60 & 88 \\
\hline Total & 58,8 & 60 & 18 & 98 \\
\hline
\end{tabular}



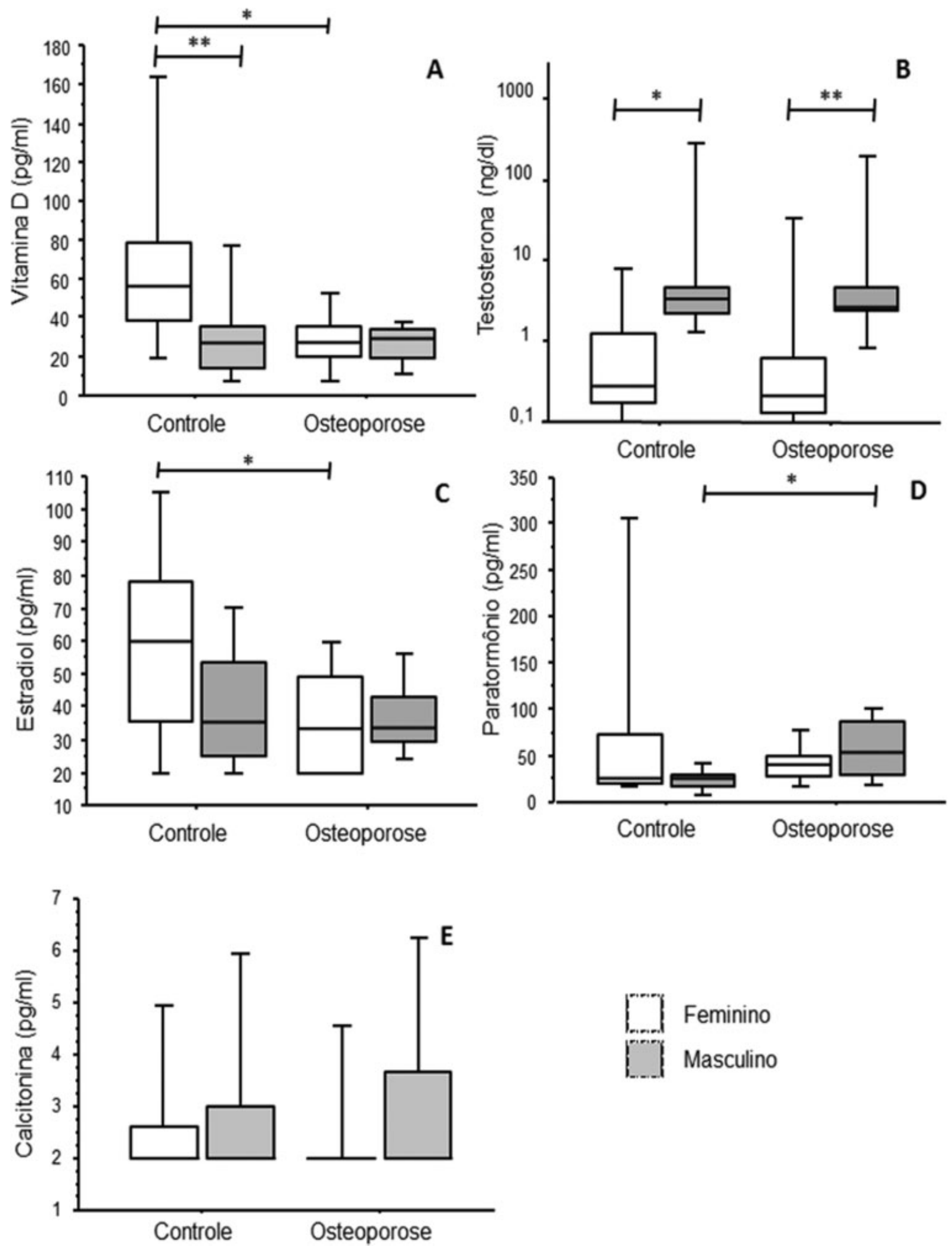

Fig. 1 Dosagem hormonal por quimiluminescência de pacientes do sexo feminino e masculino diagnosticados com osteoporose e pacientes do grupo controle. (A) Vitamina D1,25, sendo ${ }^{*} p=0,0169$ e ${ }^{* *} p=0,0275$; (B) Testosterona, sendo ${ }^{*} p=0,0023 \mathrm{e}^{* *} p=0,0046$; (C) Estradiol, sendo $p=0.047$; (D) Paratormônio, sendo $p=0.0065$; (E) Calcitonina (Mann-Whitney). 
mulheres de maior idade pode contribuir na predisposição à osteoporose. 0 presente estudo mostra também que níveis reduzidos de estradiol estão relacionados com o aparecimento da osteoporose em mulheres a partir de 60 anos, condizente com outros estudos que mostram que a saúde óssea está inversamente relacionada com níveis reduzidos de estradiol. ${ }^{7,8,14}$ A deficiência de estradiol também foi associada com a presença de osteoporose em homens $>64$ anos, ${ }^{15}$ embora essa associação não tenha sido encontrada nos resultados obtidos.

A remodelação óssea também tem sido estimulada pelo paratormônio; um estudo feito com mulheres idosas mostra que houve um aumento significativo de paratormônio em mulheres com osteoporose. ${ }^{16}$ Em nosso estudo, níveis significativamente maiores de paratormônio foram encontrados em homens com osteoporose, mostrando que a presença do paratormônio contribui para o aparecimento da doença em homens $>60$ anos, ao contrário das mulheres, que não tiveram diferenças nos níveis de paratormônio.

A testosterona não foi um fator limitante para o aparecimento da doença nos pacientes do nosso estudo; este hormônio parece estar mais relacionado com as diferenças entre os gêneros masculino e feminino. Contudo, um estudo mostra que a deficiência de testosterona em homens $>64$ anos está associada com a rápida perda óssea e, consequentemente, com o desenvolvimento de osteoporose. ${ }^{15}$ Embora dados da literatura mostrem que a calcitonina age inibindo a reabsorção óssea, ${ }^{17}$ não foram encontradas diferenças significativas nos níveis de calcitonina entre os grupos e gêneros estudados.

\section{Conclusão}

0 presente estudo sugere que mulheres com osteoporose apresentaram níveis significativamente menores de estradiol e vitamina D quando comparadas com mulheres jovens sem osteoporose. Já homens com osteoporose apresentaram níveis significativamente maiores de paratormônio quando comparados com homens sem osteoporose, o que mostra a importância dos hormônios e da vitamina D no desenvolvimento da doença. Já a testosterona, apesar de níveis menores estarem associados com a osteoporose, nosso estudo não demonstra uma associação com a doença; há uma diferença significativa apenas em relação ao gênero, independentemente da idade e da presença de osteoporose.

Contribuições dos autores

Todos os autores contribuíram para a concepção e delineamento do estudo. $O$ estudo foi concebido por Rodrigues V. e Rodrigues D. B. R. Gaia L. F. P., Sousa F. F. A., Favaro P. I., Malheiros-Souza D. coletaram o material, prepararam o banco de dados e realizaram as análises estatísticas. Malheiros-Souza D. realizarou o teste imunoenzimático (ELISA) e a citometria (CBA). Malheiros-Souza D. e Rodrigues D. B. R. redigiram o rascunho do manuscrito e o revisaram. Rodrigues $\mathrm{V}$. revisou o manuscrito.

\section{Fontes de suporte}

0 presente estudo teve apoio das instituições Universidade de Uberaba, da Universidade Federal do Triângulo Mineiro e do Centro de Educação Profissional, além do suporte financeiro da Fundação de Amparo à Pesquisa do Estado de Minas Gerais (FAPEMIG), da Coordenação de Aperfeiçoamento de Pessoal de Nível Superior (CAPES) e do Conselho Nacional de Desenvolvimento Científico e Tecnológico (CNPq).

\section{Conflito de Interesses}

Os não têm conflito de interesses a declarar.

\section{Referências}

1 Zaidi M. Skeletal remodeling in health and disease. Nat Med 2007; 13(07):791-801

2 Charles JF, Coury F, Sulyanto R, et al. The collection of NFATc1dependent transcripts in the osteoclast includes numerous genes non-essential to physiologic bone resorption. Bone 2012;51(05): 902-912

3 Udagawa N, Takahashi N, Yasuda H, et al. Osteoprotegerin produced by osteoblasts is an important regulator in osteoclast development and function. Endocrinology 2000;141(09):3478-3484

4 Breckon JJ, Papaioannou S, Kon LW, et al. Stromelysin (MMP-3) synthesis is up-regulated in estrogen-deficient mouse osteoblasts in vivo and in vitro. J Bone Miner Res 1999;14(11):1880-1890

5 Silva BC, Bilezikian JP. Parathyroid hormone: anabolic and catabolic actions on the skeleton. Curr Opin Pharmacol 2015;22:41-50

6 Damien E, Price JS, Lanyon LE. Mechanical strain stimulates osteoblast proliferation through the estrogen receptor in males as well as females. J Bone Miner Res 2000;15(11):2169-2177

7 Gui Y, Duan Z, Qiu X, et al. Multifarious effects of $17-\beta$-estradiol on apolipoprotein $\mathrm{E}$ receptors gene expression during osteoblast differentiation in vitro. Biosci Trends 2016;10(01):54-66

8 Kousteni S, Han L, Chen JR, et al. Kinase-mediated regulation of common transcription factors accounts for the bone-protective effects of sex steroids. J Clin Invest 2003;111(11):1651-1664

9 Wiren KM, Toombs AR, Semirale AA, Zhang X. Osteoblast and osteocyte apoptosis associated with androgen action in bone: requirement of increased Bax/Bcl-2 ratio. Bone 2006;38(05):637-651

10 Adams JS. Vitamin D as a defensin. J Musculoskelet Neuronal Interact 2006;6(04):344-346

11 Chen H, Gilbert LC, Lu X, et al. A new regulator of osteoclastogenesis: estrogen response element-binding protein in bone. J Bone Miner Res 2011;26(10):2537-2547

12 Weaver CM, Alexander DD, Boushey CJ, et al. Calcium plus vitamin D supplementation and risk of fractures: an updated metaanalysis from the National Osteoporosis Foundation. Osteoporos Int 2016;27(01):367-376

13 Bischoff-Ferrari HA, Conzelmann M, Dick W, Theiler R, Stähelin HB. [Effect of vitamin $D$ on muscle strength and relevance in regard to osteoporosis prevention]. Z Rheumatol 2003;62(06):518-521

14 Kousteni S, Bellido T, Plotkin LI, et al. Nongenotropic, sex-nonspecific signaling through the estrogen or androgen receptors: dissociation from transcriptional activity. Cell 2001;104(05):719-730

15 Fink HA, Ewing SK, Ensrud KE, et al. Association of testosterone and estradiol deficiency with osteoporosis and rapid bone loss in older men. J Clin Endocrinol Metab 2006;91(10):3908-3915

16 Al-Daghri NM, Aziz I, Yakout S, et al. Inflammation as a contributing factor among postmenopausal Saudi women with osteoporosis. Medicine (Baltimore) 2017;96(04):e5780

17 Martin TJ, Sims NA. Osteoclast-derived activity in the coupling of bone formation to resorption. Trends Mol Med 2005;11(02):76-81 